ARTÍCULO

\title{
Vigilar o corregir: modelos de efectividad de los derechos sociales ${ }^{1}$ Discipline or correct: models of effectiveness of social rights
}

\author{
Jesús García Cívico \\ Facultat de Ciències Jurídiques \\ Universitat Jaume I de Castelló
}

Fecha de recepción 27/12/2019| De aceptación: 21/04/2020 | De publicación: 15/06/2020

\section{RESUMEN}

Desde su concepción los derechos sociales han estado relacionados con ideas como las de dignidad, igualdad y cohesión social. Durante un tiempo sirvieron para amortiguar las diferencias económicas provocadas por el mercado, ¿qué capacidad tienen hoy para mitigar el crecimiento de la desigualdad o cuál es su relación con la inclusión social? Las aproximaciones a la eficacia de los derechos sociales han seguido tradicionalmente distintas vías, junto a índices y magnitudes relativos a la distribución de la renta, se han desarrollado indicadores de derechos sociales. La mayoría de esos modelos partían de variables características de un tipo de eficacia «ex post» obviando la importancia de la creación de desigualdades en el origen, así como una diversificación del agente responsable. Este trabajo reflexiona sobre la viabilidad de los distintos modelos de eficacia real o efectividad de los derechos sociales en relación con la desigualdad, aportando ideas para un modelo complejo que incluya elementos propios de la justicia social igualitaria y meritocrática, así como novedades relacionadas con la «Better Regulation» y los análisis de impacto normativo.

\section{PALABRAS CLAVE}

Desigualdad, derechos sociales, efectividad, mérito, impacto regulatorio

\section{ABSTRACT}

Since its conception, social rights have been related to ideas such as those of dignity, equality and social cohesion. They served to cushion the economic differences caused by the market, what capacity do they have today to mitigate the growth of inequality or what is their relationship with social inclusion? Approaches to the effectiveness of social rights have traditionally followed different routes, along with indices and magnitudes relative to income distribution, indicators of social rights have been developed. Most of these models started from variables characteristic of an "ex post" type of efficiency, ignoring the importance of creating inequalities at the source, as well as a diversification of the responsible agent. This work reflects on the viability of the different models of real efficacy or effectiveness of social rights in relation to inequality, providing ideas for a complex model that includes elements of egalitarian and meritocratic social justice, as well as news related to «Better Regulation» and regulatory impact analysis.

\section{KEY WORDS}

Inequality, Social Rights, Effectiveness, desert, Better Regulation

\footnotetext{
${ }^{1}$ Este artículo se enmarca en el proyecto Transformaciones de la justicia. Autonomía inequidad y ejercicio de derechos DER201678356-P, IP Cristina García Pascual. Igualmente, en los apartados relativos a mejora legislativa en «Aportaciones metodológicas para la evaluación del proceso legislativo y la efectividad de la regulación», DER 2016.79506-R, Ministerio de Ciencia e Innovación, IP Ignacio Aymerich Ojea.
} 
Sumario: 1. Introducción. 2. Grandes magnitudes de la desigualdad. 3. La efectividad de la vía impositiva. 4. Modelos predistributivos. 5. Modelos basados en indicadores de derechos. 6. Modelo meritocrático. 7. La efectividad de los derechos sociales y las transformaciones estructurales. 8. La eficacia de los derechos sociales, «Better Regulation» y análisis de impacto regulatorio. 9. Conclusiones. Bibliografía.

\section{Introducción}

El aumento de la desigualdad se ha convertido en uno de los principales problemas del mundo. En el seno de la Unión Europea (UE) se debate sobre la acentuación de la divergencia norte-sur² mientras que la inhabitabilidad de regiones enteras de África incrementada por el calentamiento global es un problema de máxima urgencia. Se trata, no solo de las diferencias entre regiones del planeta o entre estados, sino de las profundas desigualdades en el interior de los países. Los países del África occidental están desarrollando políticas que aumentan la distancia entre ricos y pobres según recientes informes realizados por Oxfam y por Development Finance International (DFI) ${ }^{3}$. Incluso en un país con una larga tradición de libre mercado y enorme tolerancia hacia la disparidad de rentas como EEUU, la desigualdad interna es vista como un problema. Según una reciente encuesta de The New York Times y $C B S$, un $60 \%$ de los estadounidenses opinan que su Gobierno debería hacer más para reducir la brecha entre ricos y pobres ${ }^{4}$. Autores como Atkinson, Stiglitz, Wilkison o Pickett han analizado tanto el problema de la división social que supone la existencia de altas cuotas de desigualdad económica como sus preocupantes efectos sobre los derechos. Algunos han propuesto nuevos índices y fórmulas sencillas, al estilo de Thomas Pikkety quien sustenta buena parte de su teoría sobre una fórmula matemática aparentemente simple: en el sistema capitalista actual, la tasa de retornos del capital (r) ${ }^{4}$ siempre supera a la tasa de crecimiento de renta $(\mathrm{g})$, esto es, $\mathrm{r}>\mathrm{g} .{ }^{5}$

\footnotetext{
${ }^{2}$ AYALA CAÑÓN, L., JURADO MÁlAGA, A., PÉREZ MAYO, A. J., «La lenta convergencia de España con la UE en pobreza y desigualdad», Documentación social, núm., 157, 2010, pp. 95-116.

3 OXFAM, «Informe anual de desigualdad 2019», https://www.oxfamintermon.org/es/que-hablamos-cuando-hablamos-desigualdad Consulta del 7 de febrero de 2019.

4 «A New York Times/CBS News Poll on Income Inequality and Workers' Rights». The New York Times, 3 de junio de 2015 https://www.nytimes.com/interactive/2015/06/04/business/inequality-poll.html Consulta 29 de octubre de 2019.

${ }^{5}$ Cuando se produce esta tendencia la riqueza heredada crece a una mayor velocidad que la producción y los ingresos, las personas con riqueza heredada sólo necesitan una parte de sus ingresos del capital para ver ese mismo capital crecer a un ritmo más rápido de lo que lo hace la economía en su totalidad. PIKETTY, T., Capital in the 21st Century, London: The Belknap Press of Harvard University Press, 2014, p.26.
} 
Pensemos en la mejor manera de comprender las dinámicas de la igualdad y la forma en que ésta se ve afectada por medidas provenientes de la política y el derecho. Imaginemos que, una vez asumidas como problemáticas las grandes diferencias entre regiones y estados del planeta o entre los ciudadanos de un mismo estado, quisiéramos hacer un seguimiento de la desigualdad, averiguar si tal o cual política está consiguiendo reducir la brecha. Si en el primer punto - el de la medición de la desigualdad entre estados - podría acudirse, no sin cierta polémica, a indicadores macroeconómicos como el PIB o a la cantidad de empleo creado por una economía para medir la llamada «riqueza real», en el segundo - desigualdad en el seno de la ciudadanía - se acudiría a estadísticas cuantitativas y herramientas del tipo Índice de Gini. No obstante, ambos modelos de seguimiento de la desigualdad han resultado ser problemáticos, o, al menos insuficientes. $\mathrm{Si}$, como suele decirse, el principal problema de la medición de la desigualdad entre estados es que obvia la distribución de la riqueza, en el análisis estatal quedan a menudo diluidas facetas del bienestar social, así como magnitudes relativas a la justicia social, la calidad de vida, las aspiraciones individuales, o, en lo que nos interesa aquí, la capacidad correctora de las desigualdades que tienen o pueden tener los derechos sociales.

La igualdad es un concepto complejo, un valor, un principio y una norma, su significado jurídico, social o político no se limita a ser una meta de los derechos sociales ${ }^{6}$ y lo mismo puede decirse en sentido contrario: como razonaremos después, el fin de los derechos sociales no es estrictamente la mejora de las condiciones de vida de los más desaventajados. Tampoco se justifica únicamente por la protección de la autonomía o a libertad personal. En todo caso, ese primer campo - el de la efectividad de los derechos sociales como correctores de la desigualdad - es también un tema muy amplio: va desde el modelo y funciones del Estado a los problemas de implementación más generales ${ }^{7}$, desde la percepción de la desigualdad legítima ${ }^{8}$ hasta las prestaciones relativas a la pobreza, incluso cuando

\footnotetext{
${ }^{6}$ PÉREZ LUÑO, A. E., Dimensiones de la igualdad, Madrid: Dykinson, 2005, pp. 15-18.

${ }^{7}$ CALVO GARCÍA, M., «La implementación y efectividad de los derechos económicos, sociales y culturales», en V. ZAPATERO GÓMEZ, M. I., GARRIDO GÓMEZ, (Coords.), Los derechos sociales como una exigencia de la justicia, Universidad de Alcalá: Servicio de Publicaciones, 2009, pp. 201-230.

${ }^{8}$ Un tema ya apuntado en NOYA, J., Ciudadanos ambivalentes. Actitudes ante la igualdad y el Estado de bienestar en España, Centro de Investigaciones Sociológicas, 2004. Madrid: Siglo XXI.
} 
ocurre, como en nuestro país, que no hay todavía un sistema de prestaciones asistenciales vertebrado que defina una última red de protección social más o menos homogénea. ${ }^{9}$

Tampoco la desigualdad se agota en la urgente cuestión de la pobreza o en el problema de las condiciones de vida de los sectores más desfavorecidos, sino que como magnitud dinámica apunta a tendencias que afectan a la cohesión y la estratificación social. En nuestro país, y a grandes rasgos, podemos decir que la tendencia a la desigualdad se ha mantenido incluso en épocas de bonanza económica y se ha agudizado en tiempos de crisis ${ }^{10}$. Los derechos sociales constituyen, dadas ciertas condiciones, una respuesta razonable y justa para una refundación de las bases sociales de la ciudadanía democrática $^{11}$. ¿Qué capacidad de inclusión tienen hoy? ¿Qué papel desempeñan sus exigencias y garantías que llevan aparejadas, su fuerza normativa, su lenguaje en tiempos de crisis y recortes sociales $^{12}$ ? ¿Cuál es su influencia en la estructura socio-económica o cómo afectan a esa función del estado de derecho que tiene que ver con el reparto de cargas y beneficios? ¿Son los derechos sociales una herramienta adecuada para mitigar, o, mejor, para solucionar problemas derivados de la desigualdad social? Si la respuesta es afirmativa y si la cuestión principal en este punto es su efectividad, ¿cómo medirla? Estas cuestiones son sumamente interesantes no solo para los sociólogos del derecho sino para los juristas interesados en la igualdad y en la realidad social, en la eficacia real, externa o sociológica del derecho ${ }^{13}$, y particularmente en la efectividad de los derechos sociales. Su amplitud nos ha sugerido solo esbozar en este trabajo una serie de modelos, sintetizarlos, extraer, por así decir, sus ideas-guía, destacar algunas ventajas e inconvenientes, retener algunos aspectos que podrían integrar un modelo complejo, sofisticado y no meramente coyuntural.

\footnotetext{
${ }^{9}$ AYALA CAÑÓN, L., «El gasto público en programas de lucha contra la pobreza: tendencias, determinantes y necesidades de reforma», Papeles de economía española, núm. 147, 2016, pp. 145-166.

${ }^{10}$ GARCÍA CÍVICO, J., «La (des) igualdad económica y la (in) eficacia social de las normas constitucionales sobre igualdad», Sistema: revista de ciencias sociales, núm. 237, 2015, pp. 91-112

${ }^{11}$ AÑÓN, M. J., «Derechos sociales: cuestiones de legalidad y de legitimidad», Anales de la Cátedra Francisco Suárez, núm., 44, 2010, pp.15-41.

12 CALVO GARCÍA, M., «Crisis económica y efectividad de los derechos sociales», en BERNUZ M. J., y CALVO M., (eds.), La eficacia de los derechos sociales, cit., pp. 120 y ss. Vid., CABO, C., «Teoría crítica de la democracia, en NOGUERA FERNÁNDEZ, A., (Dir.), Crisis de la democracia y nuevas formas de participación, Valencia: Tirant lo Blanch, 2013.
}

${ }^{13}$ HIERRO, L., La eficacia de las normas jurídicas, Barcelona: Ariel, 2003, p. 18. 
En general, podemos convenir en que en la práctica coexisten distintas formas de tratar la evolución de la desigualdad, aproximaciones - quizás no propiamente «modelos» dado que tras ellos no suele haber un marco teórico explícito- que se sustituyen o alternan en función de las prioridades de la investigación. Una primera impresión es que la mayor parte de los esfuerzos destinados a comprobar el grado de efectividad de los derechos sociales en relación con la igualdad miran los resultados de una serie de medidas que suelen agruparse bajo la etiqueta «ex post». Sin embargo, desde hace un tiempo, incluso antes de que el politólogo Jacob Hacker acuñara con éxito el término «predistribución», otros teóricos y actores de la política (desde la socialdemocracia nórdica a las más recientes iniciativas de Ed Miliband en Reino Unido) aconsejaban poner el énfasis en el origen de la desigualdad social, en la distribución primaria de las oportunidades y de la renta, esto es, en la estructura socio-económica, en particular en el ámbito laboral y en la regulación de los mercados (tanto económicos como financieros) en lugar de concentrarse en combatir los efectos, es decir en reaccionar ante las consecuencias de un orden de cosas previo más o menos inamovible. ¿Y no podría ocurrir, por su parte, que un modelo excesivamente centrado en indicadores de distribución de la renta olvidara algunos principios básicos de la justicia distributiva en relación con el desempeño, los incentivos y el sistema del mérito, principios que tienen algo que decir en relación con derechos sociales como los de educación y trabajo? Ni los indicadores del tipo índice de Gini, ni los indicadores típicos de derechos sociales suelen ponerse en relación con datos relativos a ese concepto - a mitad de camino entre la teoría sociológica y la justicia social - que llamamos «meritocracia», en particular a algunos de sus corolarios, como son la movilidad social vertical, la confianza, la percepción sobre la legitimidad de estatus y la igualdad de oportunidades. Hay obstáculos que impiden que la eficacia jurídica del derecho también sea una eficacia fáctica, es decir, tenga una concretización en la realidad el derecho subjetivo, por ello, como veremos, se puede hablar de modelos de indicadores de derechos. Richard Flathman proporcionaba una interesante definición sociológica general del ejercicio de los derechos que nos interesa aquí según la cual tal ejercicio es una gama de acciones orientadas normativamente y recurrente en el tiempo: Dichas acciones son entendidas por sus participantes como coincidentes en un grado significativo, e implican interacciones entre los detentadores de los derechos, el contenido de los mismos, personas con obligaciones correlativas a los derechos de los detentadores, personas en posiciones de autoridad y sus obligaciones respecto de estos derechos, y otras personas que participan de las prácticas pero que no 
ocupan ninguno de los roles antes mencionados. ${ }^{14}$ En lo que sigue, trazaremos una breve exposición de una tipología básica de las principales formas, aproximaciones o «modelos» de eficacia sociológica o real o efectividad, más allá de la alternativa entre enfoques predistributivos y distributivos. Discutiremos algunos de sus puntos fuertes y señalaremos algunas de sus principales debilidades metodológicas y sustantivas.

\section{Grandes magnitudes de la desigualdad}

Dos de las primeras y grandes magnitudes utilizadas para medir la diferencia de riqueza entre países son el Producto Interior Bruto (PIB) y la renta per cápita (el PIB dividido entre la población), ambas proporcionan información relativa a la desigualdad regional o global, pero ninguna — ni como cantidad numérica ni como media aritmética - informa sobre el reparto de la renta, esto es, sobre la cuestión de la distribución de la riqueza. Algo similar sucede con la tasa de paro: la extensión del empleo precario, y el fenómeno de los «working poors» no solo rompe con la antigua identificación entre trabajo e integración social, o entre trabajo e ingreso digno, sino que permiten que una elevada renta per cápita y una reducida tasa de paro en un determinado estado o región coexistan con elevadas tasas de pobreza y desigualdad.

Las enormes diferencias socioeconómicas que se dan en el seno de muchas sociedades tampoco quedan reflejadas en indicadores del tipo de la renta per cápita a no ser que se complementen con indicadores de desigualdad. Los índices e indicadores tradicionales de desigualdad ponen el foco en grandes magnitudes relacionadas con la distribución de la renta, en especial con el problema de los efectos de la dualización socio-económica: sistemas de quintiles, como la ratio S80/S20, el Índice de Gini, etc. La ratio S80/20 puede proporcionar información relativa a la cantidad de ingresos que la quinta parte más rica del país ha obtenido en el año de referencia en relación con la parte más pobre. El coeficiente de Gini mide la desigualdad de los ingresos en una escala de 0 (todos tienen los mismos ingresos) a 100 (todos los ingresos son para una sola persona). Las limitaciones de ambos, en lo que nos interesa aquí, es que no proporcionan información específica relativa al impacto de esa desigualdad en la estructura social ni sobre el acceso desigual a derechos fundamentales. La oficina estadística de la UE Eurostat define el $60 \%$ de la mediana nacional de los ingresos como umbral debajo del cual uno

\footnotetext{
${ }^{14}$ FLATHMAN, R., The Practice of Rights, Cambridge University Press, 1976, pp. 1-10 y 161-181.
} 
está considerado en riesgo de pobreza, pero este tipo de datos en países ricos, se suele decir, puede llegar a banalizar el problema de la pobreza. Para medir la desigualdad social, la UE se basa fundamentalmente en los datos del marco de estadísticas europeas sobre ingresos y condiciones de vida EU-SILC recogidas anualmente, en el caso de España a través de la Encuesta de Condiciones de Vida (ECV), estas últimas señales interesan a aquellos que quieran conocer la efectividad de derechos sociales como los de salud y vivienda. Este modelo elemental apunta al trazado de comparativas y a la localización de tendencias y para no abandonar por completo las primeras magnitudes que hemos mencionado, el cruce de PIB y gasto social ${ }^{15}$ también podría echar luz sobre si se usan los recursos hasta el límite de sus posibilidades, lo cual ya podría redundar, al menos en teoría, en una mayor efectividad de los derechos sociales. Para hacer frente a algunas de estas limitaciones nació el Índice de Desarrollo Humano (IDH) un indicador elaborado desde 1990 por el Programa de Naciones Unidas para del Desarrollo (PNUD), un indicador sintético de los logros medios obtenidos en las dimensiones como la esperanza de vida, los logros educativos y el nivel de vida, no obstante, una mayor atención a estos factores seguía sin atender cuestiones relativas a la incidencia de políticas de gasto social. Esto nos permite asomarnos a otro modelo muy conocido.

\section{La efectividad de la vía impositiva}

La efectividad de los derechos sociales depende de muchos factores «ex post», pero sin duda uno muy importante es la cantidad de recursos con los que cuenta una determinada administración pública. Estos provienen, sobre todo, de la recaudación a través de impuestos, de ahí que un estudio sobre la vía impositiva podría incorporarse a las comparativas y análisis cuantitativos sobre efectividad de los derechos sociales. La complejidad de los derechos sociales (con su dos facetas, «negativa» $\mathrm{y}$ prestacional) y su exigibilidad ${ }^{16}$ (frente a tesis ideológicas en sentido contrario) impiden asumir que su completa efectividad dependa de coyunturas presupuestarias, pero una de las funciones del Estado de derecho (volveremos a esto un poco más adelante) es la distribución de cargas y beneficios en una

\footnotetext{
15 La tesis de Piketty está basada en su observación de las tendencias de la tasa de retorno de los activos del capital y la tasa de crecimiento de la economía. Cuando ésta última es menor que la primera la desigualdad crece: o mientras los rendimientos del capital aumenten más que el crecimiento económico de un país se incrementará la desigualdad. PIKETTY, T., Capital in the 21st Century, cit., p. 72 y ss.

16 Aquí la literatura es extensa, vid., entre otros, PISARELLO, G., Los derechos sociales y sus garantías: elementos para una reconstrucción, Madrid: Trotta, 2007. ABRAMOVICH, V. y COURTIS, CH., Los derechos sociales en el debate democrático, Madrid: Bomarzo, 2006. ABRAMOVICH, V., AÑÓN, M. J., COURTIS, Ch., Derechos sociales. Instrucciones de uso, México: Fontamara, 2001. ABRAMOVICH, V., COURTIS Ch., Los derechos sociales como derechos exigibles, Madrid: Trotta, 2002.
} 
sociedad, por ello, junto a razones de costes de servicios públicos y prestaciones públicas, el principio de proporcionalidad impositiva - que paguen más los que más tienen - está avalado por razones de justicia distributiva, a su vez, tal justificación está relacionada con la eficiencia: el mejor impuesto es aquel que tiene un mejor efecto recaudatorio global y, al tiempo, «daña» lo menos posible y a esto se le puede llamar «eficiencia impositiva» ${ }^{17}$. En general, puede decirse que la ineficacia fiscal con sus efectos negativos sobre la cohesión social se traduce en ineficacia económica ${ }^{18}$. Piketty, Atkinson y la mayoría de economistas estudiosos de la desigualdad coinciden en que desde finales del XIX hasta la década de 1970, las sociedades avanzadas de Occidente se volvieron cada vez menos desiguales gracias a la tributación progresiva, los subsidios del gobierno para los necesitados, la provisión de servicios sociales y garantías ante situaciones de crisis, pero como observa, entre otros, Pierre Rosanvallon en $L a$ sociedad de los iguales, la trayectoria del concepto de igualdad desde «el siglo XX de la redistribución», al retroceso actual de las políticas redistributivas dibuja un declive que afecta al potencial de los derechos asociados al Estado de bienestar. Determinadas prestaciones estatales pueden amortiguar los efectos de la desigualdad, pero la eficacia de principios como los de progresividad fiscal afecta a los derechos sociales solo si previamente se han marcado determinados objetivos. Por ejemplo, si atendemos a la política comparada, desde 1980 la brecha entre ricos y pobres ha aumentado de manera alarmante en EE UU y el Reino Unido debido a un sistema de impuestos que favorece a su sector financiero. Asimismo, en la UE, el mercado único ha provocado que las grandes compañías y fortunas hayan hallado nuevos recovecos para rebajar su factura fiscal. Un modelo centrado en la eficacia fiscal de los impuestos realmente vigentes, poco o nada dice sobre otros escenarios posibles.

Recuperar o establecer una estructura de tipos más progresivas en el impuesto de la renta es una de las medidas compartidas por la mayoría de los economistas preocupados por la desigualdad, En Inequality, Atkinson proponía elevarlos al 65\% así como un ensanchamiento de la base imponible y un descuento en el impuesto personal sobre la renta en las rentas del trabajo limitado al primer tramo de ingresos. Una reforma fiscal para reducir la desigualdad tampoco debería limitarse, sin embargo, a la imposición personal sobre la renta de ahí el creciente debate sobre los impuestos que gravan el capital,

${ }^{17}$ GARCÍA AMADO, J. A., «Impuestos, igualdad y estado social», Derechos y obligaciones en el estado de derecho: actas del III Coloquio Binacional México-España / Abril Uscanga Barradas (Dir. congr.), Jesús Aquilino Fernández Suarez (dir. congr.), 2017, pp. 679-689, p. 680.

18 JUDT, T., Algo va mal, Madrid: Taurus, 2010, p. 176. 
un debate actual del que se derivan propuestas cuyo seguimiento a través de datos en el tiempo constituye otro modelo. Los estudios sobre la efectividad de la vía impositiva podrían incluir datos acerca de si y cómo las transmisiones de capital - tanto mediante herencias o inter vivos - son efectivamente gravadas con un impuesto progresivo sobre las rentas de capital acumuladas en el ciclo vital. Otras piezas de ese cuadro fiscal podrían ser la tasa Tobin, el impuesto a las transacciones financieras o la llamada «revisión del gravamen sobre la propiedad inmobiliaria» ${ }^{19}$; la importancia de la riqueza heredada vuelve a tener gran protagonismo en el siglo XXI, al poder reproducirse más rápidamente que los ahorros generados a partir de las rentas del trabajo

\section{Modelos predistributivos}

A diferencia de los modelos basados en grandes magnitudes (del tipo PIB o renta per cápita) o en la actuación «ex post» (políticas de redistribución de renta, eficacia del sistema impositivo, prestaciones vinculadas a derechos, etc.), un modelo de efectividad de los derechos sociales de tipo predistributivo acoge la idea de que la desigualdad se ve afectada también, o, sobre todo, por el diseño de las instituciones socio-económicas donde ésta se produce. Por ello parece más eficiente prevenir y atender a las transformaciones de origen, en vez de actuar con medidas redistributivas a posteriori, acciones curativas o correctoras una vez las situaciones de necesidad o desventaja ya se han producido como se hace como las prestaciones asistenciales o, en general, con la «vía impositiva» vinculada a gasto social que acabamos de ver atrás.

Hay derechos sociales como la vivienda o el derecho a disfrutar de un entorno saludable donde esto resulta extraordinariamente visible, ¿no parece más eficiente regular el precio de la vivienda, al menos, de un importante parque de viviendas antes que implantar un sistema de ayudas económicas para su adquisición? ¿No parece más razonable impedir la contaminación o el deterioro del entorno antes que dedicar recursos a su lenta recuperación? La predistribución actúa más sobre las causas que sobre las consecuencias de la desigualdad, previene en lugar de «curar» la pobreza. Se trata de acciones preventivas o ex ante, ${ }^{20}$ donde, de acuerdo con José Antonio Noguera se amplia tanto el objeto como el

\footnotetext{
${ }^{19}$ ATKINSON, A. B., Inequality. What can be done, Cambridge: Harvard University Press, 2015.

${ }^{20}$ Como ha destacado en distintos lugares Noguera, definir la predistribución con el criterio del agente, y no del objeto, tiene ventajas analíticas, pues apunta a un mecanismo identificable en la acción pública (actúa el Estado mediante impuestos y prestaciones, u obliga a actuar a otros), a diferencia de lo que ocurre cuando ponemos el foco en el eje causas vs. consecuencias de la desigualdad, pues éstas
} 
agente, de la acción distributiva ${ }^{21}$ ¿ ¿Qué acciones se incluyen o podrían incluirse en este modelo predistributivo?

En cuanto al objeto, se atiende a causas de desigualdad como la educación pública ${ }^{22}$, la formación laboral, el trabajo (empleo público, trabajo garantizado, etc.) o la construcción y acceso a vivienda pública, y como este modelo no solo mira al Estado, sino también al mercado y a otros agentes privados, otra prioridad apunta al aumento de la igualdad real en el marco de la distribución primaria de la renta a través de sindicatos y a una negociación colectiva a gran escala legalmente vinculante que pueda garantizar una menor diferencia salarial y una igualdad horizontal en las retribuciones. Aquí una cuestión central es que los recursos se asignen de la forma deseada, en vez de extraer parte de esos recursos para reasignarlos mediante políticas de gasto público

Un modelo completo de eficacia de los derechos sociales debe apuntar a otros agentes puesto que la responsabilidad de que exista pobreza y desigualdad no es siempre ni únicamente del Estado. El desarrollo histórico de los derechos sociales implica un desarrollo paralelo de los mecanismos, o de los modelos de su eficacia, así si el Estado social supuso en su día una transformación del Estado en lo tocante a sus funciones, hoy se abre una nueva perspectiva sobre las relaciones entre individuos, pequeñas y medianas empresas, corporaciones, multinacionales y grandes gestores. Y si la responsabilidad es también de los agentes sociales, como la sociedad civil, el mercado y la propia estructura jurídica familiar estos pueden modificar en sentido igualitarista sus prácticas distributivas: «Si algo nos ha enseñado la historia del Estado del bienestar es que, desplazando toda la carga de la justicia social hacia los poderes públicos, los progresos distributivos que se puedan conseguir no serán sólidos ni estables en el tiempo» ${ }^{23}$.

pueden ser empíricamente difíciles de distinguir y estar entrelazadas en procesos recurrentes y complejos. NOGUERA, J. A., Repensar las políticas sociales: predistribución e inversión social / coord. por Joseba Zalakain, Borja Barragué Calvo, 2017, pp. 207-228

${ }^{21}$ De igual manera, este modelo podría incluir las evoluciones en el funcionamiento del estado (como agente distribuidor) en el ámbito de la formación laboral, las políticas activas de empleo, la creación del empleo público y vías aún inexploradas como la renta básica universal o el capital básico). Ibidem.

${ }^{22}$ E impacto del derecho social en términos de inclusión/ cohesión social. Aquí se puede desagregar aspectos materiales, aspectos simbólicos, aspectos relativos a representación — por ejemplo, el caso de la «etnización» de las aulas, concentración población de origen inmigrante en relación con la educación como derecho social. GARCÍA CÍVICO, J., «Indicadores de integración social del inmigrante: el derecho a la educación», DE LUCAS MARTÍN, J., (ed. lit.), AÑÓN ROIG M. J., (ed. lit.), Integración y derechos: a la búsqueda de indicadores, Madrid: Icaria, 2013, pp. 37-66.

${ }^{23}$ NOGUERA, J. A., «Predistribución: ¿de qué hablamos, y por qué?», El País, 22 de julio de 2015. 
Aquí, el problema, paradójicamente viene de la propia sociedad civil que se ha mostrado reticente en muchos países a medidas como la de la limitación salarial. La tolerancia de la ciudadanía a los sueldos desorbitados de los ejecutivos (tal como se conocieron en el contexto de la crisis de Leman Brothers) ya se exacerbó durante la década de los 70 y los 80 y probablemente una de las mejores contribuciones a la comprensión psicológica y etnográfica sobre ese fenómeno fuera la de Karen Ho en Liquidated: An Ethnography of Wall Street (2009), donde describía cómo se establecen las escalas de valores a través de las cuales se eligen sueldos y compensaciones en general dentro de las grandes corporaciones. En nuestro país, Javier Noya ya expuso la tendencia dominante a considerar al mercado un medio más eficiente (y legítimo) de asignación de recursos y la profunda ambivalencia de la opinión pública a este respecto. ${ }^{24}$ Hace solo unos años, en 2013, se rechazó en Suiza la llamada «iniciativa 1:12», promovida por los representantes socialistas. La idea de que ningún directivo ganara más de 12 veces lo que el empleado peor pagado solo obtuvo el 35\% de los votos. Gran parte de los argumentos giraban en torno a ideas como la del carácter imprescindible de los grandes incentivos o la justicia retributiva a partir del principio del desempeño personal. Todo ello reconduce a modelos meritocráticos, diremos algo de ellos tras resumir primero los modelos basados en indicadores de derechos.

\section{Modelos basados en indicadores de derechos}

Los indicadores de derechos son magnitudes objetivas independientes de intereses políticos que hacen visibles aspectos de la realización de derechos, permiten hacer comparativas, evaluar procesos sociales y hacerlos operativos. De acuerdo con Aymerich, se trata de sistemas de análisis cuantitativos útiles en la medida en que ofrecen un punto de contraste, un referente sobre el que poner a prueba hipótesis sobre grado de desarrollo de los derechos, unos instrumentos que permitan comparaciones a largo y medio plazo con los que observar las tendencias evolutivas en periodos prolongados de tiempo $^{25}$. Integrado en un modelo, el indicador resulta una magnitud, estadística o no, cuantitativa o cualitativa de un concepto, basado en un análisis teórico jurídico previo - al que subyace un marco propositivo - que informa y describe el estado de una normativa determinada en relación con las

\footnotetext{
${ }^{24}$ NOYA, J., Ciudadanos ambivalentes. Actitudes ante la igualdad y el Estado de bienestar en España, cit., p. 140 y ss.

${ }^{25}$ AYMERICH, I., Sociología de los derechos humanos. Un modelo weberiano contrastado con investigaciones empiricas, cit., p. 109 y ss. BARSH, R. L., «Measuring Human Rights, Problems of Methodology and Purpose», Human Rights Quarterly, vol. 15, nº 1, 1993, pp. 87-121.
} 
acciones políticas, sociales y legislativas llevadas a cabo con miras a su efectividad. ${ }^{26}$ En lo que toca a los derechos sociales, como recordaba Manuel Calvo, el debate teórico sobre los indicadores se inició con trabajos como el Realization of Economic, Social and Cultural Rights de Danilo Türk, una línea reflejada en la Declaración de Viena de $1993^{27}$. En el ámbito del desarrollo ${ }^{28}$ o en el de los derechos sociales, se puede hacer una selección no arbitraria de datos sobre la distribución de la renta y de la riqueza, condiciones de salud y trabajo, acceso a la vivienda, educación, etc. Los derechos sociales como derechos humanos ${ }^{29}$ fundamentan la presencia de obligaciones de diversos tipos que convencionalmente se identifican como obligaciones especiales que se imponen sobre personas o instituciones determinadas, y obligaciones generales cuya responsabilidad recae sobre todos, ambas pueden ser positivas o de acción y negativas, o de omisión. ${ }^{30}$

Los indicadores estructurales apuntan al cuadro o marco normativo, a la justiciabilidad (instancias para reclamar en caso de violación: remedio y reparación), los indicadores de proceso y resultado examinarían el grado de disfrute, goce o ejercicio de los derechos sociales (de acuerdo con el molde de los derechos subjetivos) tanto la faceta «liberal» como la prestacional. Entre otros criterios que deben delinear el marco previo deben destacarse los requisitos de efectividad e igualdad de los derechos, el estándar de obligación mínima o el principio de progresividad. Un modelo que quiera dar cuenta de los efectos de los derechos sociales sobre la desigualdad debe operar con una sólida base teórica, conocer las obligaciones y garantías (también como expansión de estas, piénsese en el derecho a la salud en conexión con derecho a la vida) y los elementos que califican a derechos como el derecho a un nivel de vida adecuado. El diseño de indicadores de proceso y resultados debe tener en cuenta el significado de la progresividad de los derechos sociales, así como la prohibición de regresividad ligada

\footnotetext{
${ }^{26}$ «A piece of information used in measuring the extent to which a legal right is being fulfilled or enjoyed in a given situation», GREEN, M., «What We Talk about when We Talk about Indicators: Current Approaches to Human Rights Measurement», Human Rights Quarterly, vol. 23, 2001, pp. 1062-1097, p. 1062.

${ }^{27}$ CALVO GARCÍA, «La implementación y efectividad de los derechos económicos, sociales y culturales», cit., p. 217.

${ }^{28}$ Comisión Interamericana de Derechos Humanos, «Lineamientos para la elaboración de indicadores de progreso en materia de derechos económicos, sociales y culturales», OEA/ser/L/V/II.129, 5 de octubre de 2007. Informes del Observatorio de política social y derechos humanos

${ }^{29}$ Datos que a través de la información que facilitan permiten conocer la situación, implementación, grado de cumplimiento, etc. en términos de derechos humanos. En palabras de Richard P. Claude, un análisis global asistido por técnicas estadísticas que puede ser útil no sólo para propósitos científicos o académicos sino también para fortalecer la definición de las públicas. JABINE, Th. CLAUDE, R. (eds.), Human Rights and Statistics. Getting the record straight, University of Pennsylvania Press, Philadelphia, 1992 , p. 5.
}

${ }^{30}$ AÑÓN ROIG, M. J., «Derechos humanos y obligaciones positivas», cit., p. 43. 
al principio de constitucionalidad ${ }^{31} \mathrm{y}$, más ampliamente, al valor de la constitución como límite normativo al poder. A menudo sucede que la cláusula del Estado social, que se inserta en este complejo de valores, es seriamente puesta en cuestión en el caso de la regresión de derechos sociales, de modo que la supresión de los derechos y/o el retroceso de su tutela no solo podría suponer una arbitrariedad, sino también una auténtica vulneración del contenido del modelo de Estado social y democrático de derecho lo cual es sensible a indicadores estructurales.

La integración y la cohesión social son procesos dinámicos que dependen tanto del marco jurídico como, en gran medida, de la voluntad política, cuestiones todas susceptibles de evaluarse mediante los tres tipos de indicadores ${ }^{32}$. En los índices de derechos humanos se privilegia a menudo a los indicadores de derechos civiles y políticos, pero a la mayor o menor presencia de indicadores de determinados derechos subyace una opción política que afecta a la investigación empírica. Esto sucede paradigmáticamente en el tradicional descuido de los derechos económicos y sociales. Son conocidas las reticencias por un sector de la doctrina a esta categoría de derechos (exigibilidad menor, «de segundo grado», condicionada a recursos, etc.) que los considera promesas de corte político, a lo sumo derechos incompletos o categorías difícilmente judiciables, no compartimos ninguna de éstas y no hay ninguna razón para no incluir los derechos económicos y sociales en el sistema global sobre efectividad de los derechos humanos más allá de diferencias en la concreta cuestión de la metodología. Un subapartado apunta a la igualdad y los derechos sociales (educación, salud, vivienda, etc.) en el cruce con las medidas predistributivas vistas antes (es el caso de las grandes corporaciones y otras empresas privadas y el uso de poder económico privado en problemas como el acaparamiento de tierras, agresiones al medio ambiente, expulsión poblacional por construcción de obra pública y otras).

En el amplio ámbito de los derechos humanos, la prohibición de regresividad es un punto estable para la evaluación de la efectividad de los derechos sociales, tanto en lo que toca al principio de convencionalidad entendido en un sentido amplio como en lo relativo a la interpretación de los tratados internacionales, concretamente, en la exégesis doctrinal del Comité de Derechos Económicos, Sociales

\footnotetext{
${ }^{31}$ AÑÓN ROIG, M. J., «Derechos humanos y deberes: Efectividad y prohibición de regresividad», Repensar los Derechos Humanos / Angeles Ródenas (ed. lit.), 2018, pp. 263-305, p. 268 y ss.

${ }^{32}$ Vid, los trabajos contenidos en AÑÓN ROIG, Mª. J., DE LUCAS, J., Integración y derechos: a la búsqueda de indicadores, cit., GARCÍA CÍVICO, J., La integración social del inmigrante a través del Derecho: Hacia un sistema de indicadores, Cuadernos de Democracia y Derechos Humanos N 4, Universidad Alcalá-Defensor del Pueblo, 2011.
} 
y Culturales (DESC), órgano encargado de supervisar el cumplimiento del Pacto Internacional de Derechos Económicos, Sociales y Culturales (PIDESC). El principio de no regresividad significa la prescripción de no adoptar medidas, no aprobar normas jurídicas o no implementar políticas públicas que empeoren sin justificación la situación de los derechos sociales ${ }^{33}$. Los indicadores forman parte de un sistema de información, pero también forman parte de un marco teórico. Los indicadores son necesarios en el diseño específico de cómo poner en contacto conceptos y teorías con hechos empíricos tratando de encontrar correlatos que permitan entender el comportamiento en la práctica de variables que han de ver con hipótesis, con la teoría sobre bajo qué condiciones podemos decir que un derecho social es eficaz. ${ }^{34}$

\section{Modelo meritocrático}

Los modelos meritocráticos observan la desigualdad desde el punto de vista de la estratificación social. La movilidad social vertical debida a desempeños personales, educacionales, profesionales laborales etc. es un indicador de su eficacia. Aquí otras señales vendrían dadas por corolarios de la meritocracia como son la apertura de cargos y posiciones sin discriminación formal, la reducción del impacto de la herencia y el «capital social»o los avances de una igualdad de oportunidades no meramente retórica sino acompañada de medidas que "corrigen" la metafórica "pista de salida".

Como está quedando de manifiesto, un modelo de efectividad de los derechos sociales es siempre un modelo complejo, no puede limitarse a las grandes magnitudes numéricas ni a los indicadores tradicionales, tampoco debe centrarse acríticamente en medidas «ex post». Vimos precisamente al hilo de estas que la regulación de la educación incide en la desigualdad futura, de forma que un modelo de efectividad de los derechos sociales que aceptara el paradigma predistributivo existente debería ser sensible a modificaciones estructurales, ¿no parece también razonable corregir las desigualdades que

\footnotetext{
${ }^{33}$ SEPÚLVEDA, M., «La interpretación del Comité de Derechos Económicos, Sociales y Culturales de la expresión "progresivamente”", en COURTIS Ch. (Comp.), Ni un paso atrás. La prohibición de regresividad en materia de derechos sociales, CEDAL-CELS, Buenos Aires, 2006, pp. 117-152. La regresividad normativa se determina a través de un análisis comparativo de la nueva regulación y la norma que esta ha modificado a fin de evaluar si la norma posterior suprime, limita o restringe derechos o beneficios reconocidos por la anterior. AÑÓN, M. J., «¿Hay límites a la regresividad de los derechos sociales?», Derechos y libertades, núm. 34, 2016, pp. 82-83, p. 63.

${ }^{34}$ Desde la prioridad concedida a la eficacia real, esta reflexión teórica se completa con el estudio de las condiciones sociohistóricas, culturales y económicas que se traducen en mayor estabilidad y efectividad de los derechos. AYMERICH, I., Sociología de los derechos humanos. Un modelo weberiano contrastado con investigaciones empíricas, cit. p. 17 y ss.
} 
redundan en estancamientos sociales, escasa movilidad social, etc. y regular la influencia de los factores que impiden una distribución salarial vinculada realmente al desempeño, al talento, a la inversión personal y al esfuerzo?

Hoy en día, entendemos por mérito personal la aptitud reflejada en una serie de títulos académicos que acreditan la capacidad (o la mejor capacidad) para ocupar una plaza en la administración o un cargo público. Comprendido así, el mérito es un principio de selección más racional, abierto y con mucha probabilidad, más justo que el que resulta de las tradicionales influencias del poder político, la endogamia propia de las clases sociales, las redes clientelares o el nepotismo. También se entiende, ya en el ámbito privado, que el mérito individual como suma de inteligencia y esfuerzo está detrás del «éxito» o del «fracaso», en términos económicos o materiales, de una empresa o un individuo.

Es cierto que el mérito es un principio superador del nepotismo, sin embargo, el espectro de versiones de la meritocracia es demasiado variado como para permitir un consenso sobre sus indicadores. Además, el mérito es un principio perfectible que debe ponerse en relación con determinadas exigencias del principio de igualdad. La realidad muestra que el azar, las redes sociales y un buen número de variables informales inciden en la composición de la «pirámide meritocrática». A la vez, un modelo meritocrático ingenuo certificaría la ausencia de no discriminación y apertura formal de cargos como presupuestos legitimadores de los resultados de estatus. Si el acceso a la función pública bajo el principio de la capacidad y el mérito está más o menos conciliado con las implicaciones del principio de la igualdad, el ámbito privado, es posible convenir en que la legitimidad de las recompensas económicas debería estar al menos supeditada al cumplimiento de una serie de premisas más allá de la ausencia de discriminación en la contratación y en la promoción, por ejemplo, algún corrector de la excesiva influencia del capital social y familiar.

Un número dado de casos de corrupción, privilegios injustificados y de nepotismo puede afectar a la percepción de la legitimidad de la desigualdad de estatus. De igual forma, lo puede hacer la existencia de grupos en desventaja inicial e individuos excluidos en la entrada del «ascensor social» (piénsese en las personas en situación de miseria, discapacitados, etni-estratificación de nacionales de terceros países, homologación de títulos extranjeros, etc.). Si se defiende que la igualdad de oportunidades como premisa del mérito debe ser real, es decir, que debe actuarse sobre las condiciones 
del punto de partida a través de políticas redistributivas de la riqueza e integradoras de grupos tradicionalmente excluidos, entonces deberían incluirse en el modelo de efectividad de los derechos sociales medidas de tipo corrector. El concepto sociológico de igualdad de oportunidades apunta a los ingresos de unos individuos, según el nivel de estudio de los padres. Si tuviéramos que plantearlo muy brevemente, podríamos decir que si los individuos cuyos padres tienen un nivel de estudio A obtienen sueldos siempre inferiores a los individuos cuyos padres tienen un nivel de estudio B, hay desigualdad de oportunidades. Sobre este punto, los últimos informes sobre igualdad destacan que las personas nacidas en hogares pobres necesitan más de tres generaciones para ascender social y económicamente, mientras que las personas nacidas en hogares privilegiados tienen la vida asegurada más de cuatro generaciones, solo merced a su origen afortunado ${ }^{35}$. La movilidad social puesta en relación con la desigualdad social y la estructura de clases es a su vez un vastísimo tópico de la sociología. La medición de la movilidad social es un buen indicador de la efectividad de derecho como los de educación: en 2018, el $82 \%$ de la riqueza generada fue a manos del 1\% más rico, mientras que la riqueza del $50 \%$ más pobre no aumentó lo más mínimo. La riqueza extrema de unos pocos se erige sobre el trabajo peligroso y mal remunerado de una mayoría. Mientras las mujeres ocupan mayoritariamente los empleos más precarios, prácticamente todos los súper ricos son varones. Hay que recordar que con semejantes frentes abiertos y con el limitado efecto de la educación como instrumento de mejora de las posiciones sociales (la esperanza en la educación como «ascensor social»), la crisis financiera desveló la ausencia de méritos de los gestores que se enriquecieron de forma obscena, así como la necesidad de una meritocracia real ligada al talento y la profesionalidad. Las reformas del sistema meritocrático deben prestar atención a grupos desaventajados: el problema de la infrarepresentación de la mujer y la cuestión de la acción afirmativa o las medidas de discriminación inversa deben ser incluidos en el debate sobre la confección de un sistema basado en el mérito. Hoy es posible conciliar la defensa de la idea de que los puestos se ocupen por méritos con medidas como las de «acomodo razonable», cuotas, reserva de plazas a fin de que toda la sociedad pueda estar representada, por ejemplo, en atención a minorías culturales. Un breve diagnóstico sobre la fase actual de los sistemas meritocráticos - tanto en el plano práctico como simbólico - debe reparar en las tendencias de superfluidad del desempeño en el actual modelo de mercado. Las «finanzas de casino», la

${ }^{35}$ GARCÍA CÍVICO, J., «El principio de mérito a la luz de los hechos sociales», Anuario de Filosofía del derecho, 30, 2014, pp. 191216.

CEFD Número 42 (2020) I ISSN: 1138-9877 I DOI: 10.7203/CEFD.42.16694 
especulación, las redes sociales de influencia, la proximidad al poder político etc., están detrás de muchas fortunas. Al mismo tiempo, las crisis económicas son «pagadas» por personas que no son responsables de ellas, la movilidad generacional se ha interrumpido, las generaciones de nacidos a principio del siglo XXI tienen pocas posibilidades de mejorar la condición en la que nacieron, la pobreza se perpetua, todo eso supone una quiebra de los ideales meritocráticos. Cada vez son mayores las dificultades de medición real del mérito personal (por ejemplo, la valía personal aislada de factores exógenos como la red social) o de medición de la productividad dada la robotización y el aumento del subempleo fragmentado, así mismo, la vinculación del sistema prestacional con el trabajo como base meritocrática está conduciendo a una intensificación de la individualización de las protecciones sociales y a la exclusión de sectores no integrados en la sociedad salarial. Una renta básica universal, tanto en la justificación ya clásica de Philippe Van Parijs apoyada en la «libertad real» como en la idea republicana de Daniel Raventós, («la propiedad de las elecciones vitales» o el «auto-gobierno» respectivamente) podría sortear en el futuro muchos de esos problemas.

\section{La efectividad de los derechos sociales y las transformaciones estructurales}

Las estructuras de poder que se legitiman y que forman el engranaje de la sociedad que hemos apuntado en el modelo anterior no conforman lógicas «naturales», sino construidas, y al igual que podemos hablar de una estructura de la desigualdad, de mecanismos sistemáticos que dan lugar a dichas disparidades a nivel nacional o supraestatal, podemos plantear reformas estructurales que incidan a medio plazo en una mayor eficacia de los derechos sociales en atención a la igualdad. Piketty ha explicado convincentemente que la desigualdad de sueldos en diferentes países y periodos de tiempo no puede explicarse solamente en términos de productividad marginal, sino que las instituciones y las normas que regulan el mercado laboral tienen un peso significativo en los resultados. ${ }^{36}$ Atribuye la desigualdad de ingresos particulares en parte a las leyes econométricas de la productividad marginal y a las inversiones en educación y tecnología (en cuanto a la evolución de los sueldos a largo plazo), pero también al nacimiento de una cultura permisible con las jerarquías dentro de empresas y corporaciones.

${ }^{36}$ PIKETTY, T., Capital in the 21st Century, cit., p. 308 y ss. 
Frente a esto, un modelo de efectividad de los derechos sociales debe ser coherente con los principios y valores del Estado social, con la democracia ${ }^{37}$ y con el sistema jurídico (estatal y supraestatal) del que forman parte, entre las que se encuentra tanto la obligación que tienen los poderes públicos de remover los obstáculos que impiden la igualdad real como de lograr la cohesión social. Igualmente debe preguntarse por los sistemas de garantías, en el conocido modelo garantista de Ferrajoli técnicas para reducir la distancia estructural entre normatividad y efectividad, y, por tanto, para posibilitar la eficacia de los derechos fundamentales en coherencia con su estipulación constitucional $^{38}$. Como es sabido, con la aprobación de la Constitución española de 1978 se introdujeron en el texto constitucional los principales elementos normativos propios del Estado social: la cláusula de Estado social, la Constitución económica, los derechos sociales, la regulación del trabajo, etc. ${ }^{39}$ Todo ello forma parte de una estructura socio-política, no solo de un sistema jurídico, así, de acuerdo con el artículo 40. $1 \mathrm{CE}$, los poderes públicos deben promover «las condiciones favorables para el progreso social y económico y para una distribución de la renta regional y personal más equitativa». El artículo $50 \mathrm{CE}$, por su parte, afirma que los poderes públicos «promoverán el bienestar de los ciudadanos mediante un sistema de servicios sociales que atenderán sus problemas específicos de salud, vivienda, cultura y ocio». Sin embargo, la ubicación del contenido social entre los «Principios rectores» sin las garantías explícitas de los derechos fundamentales del artículo 15 al 29, el contexto de crisis europea del estado de bienestar ha dificultado tradicionalmente el diseño de un sistema de garantías robusto, así mismo, un concepto complejo, y en cierta medida ambiguo, como el de Estado social admite una pluralidad de enfoques: si se trata de un equilibrio entre capital y trabajo, un cuadro de efectividad de los derechos sociales reconocidos en su marco apunta a una estabilización real de fuerzas, en el sentido en que los intereses son efectivamente contrarrestados o armonizados. Si asumimos que el Estado debe convertirse en la instancia de mediación y garantía de los acuerdos que se establecen entre grandes organizaciones ${ }^{40}$, la eficacia global se mediría en función de la ordenación del conflicto y lo mismo ocurre si el modelo de Estado social es pensado como extensión de la representación sindical, como institucionalización de los grupos de presión o como garantía de una

\footnotetext{
${ }^{37}$ SUSÍN BETRÁN, R., «Derechos sociales-trabajo-ciudadanía-democracia. Una relación necesaria (y conveniente)”», en BERNUZ M. J., CALVO M., (eds.), La eficacia de los derechos sociales, cit. pp. 163-194.

${ }^{38}$ FERRAJOLI, L., Derechos y garantías. La ley del más débil, Madrid: Trotta, 1999, pp. 15-35.

${ }^{39}$ NOGUERA, A., «El estado social y el tratamiento de los derechos sociales en la Constitución española de 1978», Social», cit., p. 198.

${ }^{40}$ BOBBIO, N., El futuro de la democracia, Barcelona: Plaza y Janés, 1985.
} 
serie de demandas sociales que deben hacer frente a un mercado global en el que compiten países con niveles muy distintos de protección social. Como decíamos al comenzar, los derechos sociales no tienen una única fundamentación, el fin de los derechos sociales no es estrictamente la mejora de las condiciones de vida de los más desaventajados. Derechos sociales como la educación o la salud son universales y no todos sus detentadores están en situación de desventaja social. Los indicadores de derechos sociales no pueden ser los mismos que los que se utilizan para medir la evolución de la desigualdad. Los derechos sociales protegen a los individuos en ámbitos como la educación, la salud o el empleo y están estrechamente relacionados con derechos civiles como los de libertad de expresión o el libre desarrollo de la personalidad. Hace años que los desarrollos jurisprudenciales y analíticos sobre el carácter unitario de las obligaciones derivadas de los derechos humanos ${ }^{41}$ vienen reafirmando la indivisibilidad e interdependencia de los mismos lo que implica que los derechos sociales también están conectados con valores como los de libertad ${ }^{42}$.

Como ha señalado Añón, la tesis de la indisponibilidad negativa de los derechos encuentra su anclaje justificativo en todo un conjunto de principios que forman parte de un diseño institucional: el valor normativo de la constitución y la vinculación de todos los poderes a la misma, incluido el legislador (art. 9.1 CE), la efectividad del texto constitucional en una materia que forma parte del contenido de la cláusula del Estado social y democrático de derecho, el respeto a la dignidad humana y el libre desarrollo de la personalidad (10.1 CE) y la apertura al derecho internacional de los derechos humanos ${ }^{43}$. En nuestro sistema jurídico hay criterios de ámbito constitucional -constitución y bloque de constitucionalidad- que tienen carácter claramente supralegal y que pueden establecer en un plano estructural una barrera infranqueable -un nivel mínimo de prestación-para el legislador en materia de servicios fundamentales aplicables en todo el territorio ${ }^{44}$. El artículo 9.2 compromete la acción de los poderes públicos, a fin de que pueda alcanzarse la igualdad sustancial entre los individuos, con

${ }^{41}$ La efectividad de los derechos no es un desiderátum sino una obligación normativa que vincula a los estados. En el caso de los derechos sociales, el artículo 2.1 del PIDESC establece obligaciones como la de adoptar medida hasta el máximo de los recursos de los que se disponga, para lograr progresivamente, por todos los medios apropiados, inclusive en particular la adopción de medidas legislativas, la plena efectividad de los derechos aquí reconocidos. GARCÍA CÍVICO, J., «¿Qué es un indicador de derechos humanos y cómo se utiliza?», Derechos y libertades, núm., 24, 2011, pp. 179-219.

42 ANSUÁTEGUI, J., «Los derechos sociales en tiempo de crisis. Algunas cuestiones sobre su fundamentación», en BERNUZ M. J., y CALVO M., (eds.), La eficacia de los derechos sociales, Valencia: Tirant Lo Blanch, 2013, p. 31 y ss.

${ }^{43}$ AÑÓN, M. J., «¿Hay límites a la regresividad de los derechos sociales?», cit., p. 67 y 68.

${ }^{44}$ Ibid., p. 74.

CEFD Número 42 (2020) I ISSN: 1138-9877 I DOI: 10.7203/CEFD.42.16694 
independencia de su situación social (STC 39/1986, de 31 de marzo); además, el artículo 9.2 puede imponer, como consideración de principio, la adopción de normas especiales que tiendan a corregir los efectos dispares que, en orden al disfrute de bienes garantizados por la Constitución, se sigan de la aplicación de disposiciones generales en una sociedad cuyas desigualdades radicales han sido negativamente enjuiciadas por la propia norma fundamental (STC 19/1988, de 16 de febrero) ${ }^{45}$. Un modelo de efectividad de los derechos sociales debe ser sensible y crítico con retrocesos como el que supuso la implementación de políticas neoliberales en la gestión de la crisis financiera, del tipo que en nuestro país significó la reforma constitucional del techo de gasto público.

Sobre los agentes privados, hemos mencionado ya que gran parte de las medidas que caen del lado de la predistribución suponen transformaciones de tipo estructural, adicionalmente podríamos incluir en un modelo de eficacia de los derechos sociales más complejo, aquellas que partan o asuman la idea de que los derechos sociales y económicos pueden ser instrumentos de desmercantilización de las relaciones sociales. ${ }^{46}$ Coincido con Añón en que los derechos tienen prioridad normativa sobre otros objetivos económicos sociales, criterios basados en la utilidad, creencias religiosas o cuestiones ligadas al perfeccionamiento de los individuos y que tal prevalencia descansa en la consideración de que los derechos están destinados a proteger y promover intereses humanos vitales, perentorios o de importancia fundamental y en que son condición de posibilidad del propio sistema democrático ${ }^{47}$. También hay pensadores que llevan tiempo denunciando que la forma en que el capitalismo se apropia de la riqueza tiene que ver con una subversión a nivel mundial pero también al nivel de los Estados de derecho. A nivel mundial, el proceso de desposesión, acumulación y explotación proyecta un grado de violencia vertical propio de la colonización; a nivel estatal hay un encarnizamiento en las prácticas de acumulación de beneficio privado sobre los valores sociales. Entrarían de nuevo aquí, medidas de tipo predistributivo como la participación en acciones como parte de la retribución de los trabajadores. En ocasiones, el problema no es la propiedad sino la gestión de la propiedad: los responsables no son los grandes propietarios sino los grandes gestores. El desdibujamiento de los actores potenciales de la desigualdad es una señal más de que la intersección entre los derechos sociales y las dinámicas de

45 GARCÍA CÍVICO, J., «La (des) igualdad económica y la (in) eficacia social de las normas constitucionales sobre igualdad», cit., p. 101.

${ }^{46}$ LEMA AÑÓN, C., «Derechos sociales, ¿para quién?: sobre la universidad de los derechos sociales», Derechos y libertades: Revista del Instituto Bartolomé de las Casas, núm. 22, 2010, pp. 179-203.

${ }^{47}$ AÑÓN, M. J., «¿Hay límites a la regresividad de los derechos sociales?», cit., p. 77 y ss. 
desigualdad está en continua transformación, lo mismo ocurre con las demandas sociales características del Estado social que se centraron inicialmente en la extensión de la educación, la seguridad social, el empleo y la libertad sindical y la sanidad públicas ${ }^{48}$ y hoy afectan a preocupaciones en el ámbito de la conciliación de la vida familiar, personal y laboral en la empresa privada, a las necesidades de personas con discapacidad o dependencia, al agua o al medioambiente.

\section{La eficacia de los derechos sociales, «Better Regulation» $\mathbf{y}$ análisis de impacto regulatorio}

Por último, el análisis de impacto es una de las herramientas de la mejora legislativa («Better Regulation», «Regulatory Reform» o «Smart Regulation»), un concepto, hoy todavía en desarrollo, que apunta tanto a la eficacia normativa y el control del gasto, como a una serie de principios de actuación en la intervención pública para garantizar que se cumplen los objetivos de racionalidad y efectividad económica (reduciendo al mínimo el posible impacto negativo de la intervención pública) de forma respetuosa con una serie de valores y principios, así, el cuidado medioambiental, o la igualdad de género $^{49}$. Se trata de instrumentos de evaluación sistemática de los efectos de las normas y por ello nada impide que más allá del análisis coste-beneficio, los análisis de impacto se centren en los efectos de la legislación ya no, o ya no solo sobre el medioambiente y la igualdad de género ${ }^{50}$, sino sobre la desigualdad socio-económica.

El análisis del efecto de la ley o de la norma jurídica sobre sus destinatarios no es sólo el estudio de le efectividad (con fines, por ejemplo, de seguimiento de su impacto real, expresión aquí, casi equivalente a la de eficacia real, externa o sociológica), monitoreo, rendición de cuentas, etc., sino el análisis acerca de si la norma contiene en su redacción los instrumentos destinados a garantizarla (garantías, sanciones y mecanismos de ejecución de la sanción, etc.), respondiendo, así, a la

\footnotetext{
${ }^{48}$ ASENSI SABATER, J., «Origen e historia del Estado Social», Lecciones sobre Estado Social y Derechos Sociales / Albert NOGUERA FERNÁNDEZ A., (dir.), GUAMÁN HERNÁNDEZ A., (dir.), Valencia: Tirant lo Blanch, 2014, pp. 25-39.

49 AUBY, J. B., PERROUD, T. (Eds.), La evaluación de Impacto Regulatorio, Sevilla; Instituto Nacional de Administración Pública y Global Law Press, 2013. KIRKPATRICK C., PARKER D., Regulatory Impact Assessment Towards Better Regulation?, Chentelham: Edward Eldar Publishing, 2007.

${ }^{50}$ CARCELLER, G., «Análisis de impacto normativo. Proceso y metodología en perspectiva autonómica, Ética, transparencia buen gobierno y sistema electoral, Valencia: Tirant lo Blanch, 2019, pp.97-142.
} 
preocupación por su grado de cumplimiento o por la consecución de sus objetivos con un sistema de retroalimentación y evaluación de resultados..$^{51}$

Paralelamente, el estado debe evaluar las leyes y los reglamentos para determinar si lograrán beneficios públicos, si alcanzarán y cómo sus objetivos y cómo afectan a otros derechos. Entre esos efectos podría considerarse el impacto sobre la distribución de la renta, el reparto de la propiedad, la acumulación de capital en manos privadas o si tal o cual norma afecta y cómo al mercado de trabajo, a las condiciones laborales o al precio de la vivienda.

En un plano propositivo parece aconsejable una mejora de la legislación en aras de la integración en esta de mecanismos de supervisión, por ejemplo, en el mismo articulado, relativos a compromisos temporales de evaluación. A tenor de lo visto en todos los epígrafes precedentes, un instrumento así daría cuenta de una serie de obligaciones positivas que podrían aplicarse en materia de igualdad y derechos sociales. Si queremos proponer una serie de medidas relativas dirigidas a mejorar los análisis impacto legislativo a través de la introducción del análisis relativo a derechos fundamentales cabe profundizar en ellos y distinguir entre sus formatos y dimensiones. Se trata de modelos que incorporarían no solo un sistema de retro-alimentación exigente sobre la eficacia real o efectividad de los derechos contenidos en la ley sino un análisis previo de su impacto en la igualdad o en la cohesión social. Los análisis de impacto en la igualdad socioeconómica no solo serían una obligación legislativa más o menos formal sino un medio de control parlamentario del cumplimiento de compromisos políticos centrada en la eficacia real de las normas.

Por último, la evaluación de normas (o de la política legislativa) y de sus consecuencias «ex ante» o «ex post» debería asumir que existe una relación directa entre los distintos momentos de la «mejora»: por ejemplo, la evaluación «ex ante» permite fijar los términos a evaluar en la segunda (el cumplimiento de las previsiones recogidas en la memoria de impacto) y la segunda, permite mejorar los sistemas de evaluación del impacto prospectivo ${ }^{52}$.

\footnotetext{
${ }^{51}$ AYMERICH, I., «Una contribución para el buen gobierno: sobre las posibilidades de incorporar el análisis de impacto regulatorio al proceso legislativo valenciano, Ética, transparencia, buen gobierno y sistema electoral. Propuestas de mejora de la normativa valenciana, cit., p. 37.

52 Vid., MARCOS, F., «Calidad de las normas jurídicas y estudios de impacto normativo», Revista de Administración Pública, nº. 179, mayo-agosto 2009, p. 336.
} 


\section{Conclusiones}

En la génesis de los derechos sociales, el artículo 151 de la Constitución de Weimar establecía que «el orden de vida económica debe responder a los principios de la justicia con el objetivo de garantizar a todos una existencia digna», desde su concepción los derechos sociales han estado relacionados con ideas como las de dignidad y cohesión social, así que también es lícito articular modelos de efectividad de los derechos sociales que tengan en cuenta esos objetivos, de forma paralela a cómo, indicadores relacionados con la igualdad de oportunidades o el desarrollo de capacidades (en la clásica tesis de Amartya Sen) podrían sustituir a variables del tipo PIB o renta per cápita. Cuanto mayor es la distancia entre la minoría acomodada y la masa empobrecida, más se agravan los problemas sociales. Los derechos sociales son una herramienta para la igualdad (para la igualdad es aspectos sustanciales, por ejemplo, la satisfacción de necesidades, el acceso a la riqueza y las oportunidades) pero también para la libertad, la autonomía y el desarrollo individual, por ello, los modelos de eficacia de los derechos sociales orientados a la reducción o amortiguación de la pobreza son insuficientes. No importa lo rico que sea un país, sino lo desigual que sea, por ello, un modelo de efectividad de los derechos sociales tampoco debe mirar solo a los grupos desaventajados, o a la importante cuestión de la pobreza, sino de la desigualdad. De acuerdo con los informes de la OCDE, desde mediados de los noventa las políticas correctoras como los impuestos y los servicios sociales han perdido poder de redistribución de riqueza y de corrección de la desigualdad de oportunidades por ello hemos dedicado un espacio a considerar modelos basados en la eficacia fiscal. El impacto de la crisis financiera y económica y las políticas públicas que las gestionaron han afectado negativamente a la situación de la igualdad de oportunidades de acuerdo con los principales indicadores que atienden a tanto a la predistribución como a la redistribución, como la movilidad social. Nada impide que surjan modelos de efectividad de los derechos sociales en relación con su impacto en la desigualdad social basados en el incremento de las rentas del capital en detrimento de las rentas del trabajo igual que amplían el abanico de modelos aquellos que pusieran el foco sobre las dinámicas de desigualdad en términos generacionales, en porcentajes relativos a la propiedad del capital (porcentajes de acumulación de capital privado frente a fuerza de trabajo) o en las relaciones entre centro y periferia.

Un modelo que pretenda aproximarse a la efectividad de los derechos sociales debe haber respondido antes cómo considera realizado ese vasto conjunto de pretensiones y facultadas legítimas, o, 
indirectamente qué valores, qué bienes protegen. Derechos como la salud o la educación permiten aproximaciones muy distintas, podemos poner el acento en el individuo, en el porcentaje que accede y termina la educación obligatoria, o que se beneficia de la cobertura sanitaria, o bien podemos inquirir en la forma que la educación corrige desigualdades socio-económicas previas, si es realmente la vía para sociedades de movilidad social basada en el mérito o si, en relación con la salud, y a pesar de los porcentajes, se mantiene un estructura tal que la riqueza se traduce en un acceso a seguros privados y a una esperanza de vida mayor. Paralelamente al abandono de modelos con una fuerte carga ideológica, por ejemplo, los modelos marxistas basados en la emancipación y la lucha de clases, el principio del mérito aparece todavía de forma intermitente como contrapeso teórico frente a la presión impositiva dirigida a las grandes fortunas y patrimonios, pero, su vez, el desmantelamiento progresivo del sistema de protección del estado social saca del «juego meritocrático» a altos porcentajes de la población de forma análoga a cómo el manejo del capital financiero se superpone a las diferencias de desempeño, talento o esfuerzo individual. Hemos aludido así a modelos que tienen en cuenta la difusión del conocimiento y la mejora de la educación como el factor que más ha contribuido a la reducción de la desigualdad a la larga, y a la vez como factor sensible a las desigualdades de origen y capital social o heredado,

¿Es mejor vigilar el nacimiento de las desigualdades o corregirlas? Los modelos más complejos parten de la idea de que los derechos sociales presentan distintos niveles que podríamos resumir en tres: pre-distributivo, redistributivo y transformadoras de las estructuras de desigualdad. Aisladamente considerados, los modelos redistributivos son insuficientes, se requiere un modelo que distinga entre facetas o dimensiones del derecho social, su contenido esencial, su faceta prestacional, su faceta «liberal» tanto «ex ante» como «ex post», (acciones negativas, como el respeto frente a terceros, la protección ante su conculcación por acciones u omisiones de particulares privados y administración pública por abstención, no intervención de particulares privados y administración pública), un modelo que incluya medidas pre-distributivas y estructurales en relación con derechos como los de trabajo, educación y salud. Como señalaba Noguera, la justicia social no sólo es cuestión de los poderes públicos, sino que depende del conjunto de actores e instituciones, formales o informales, que asignan recursos económicos en nuestra sociedad. La integración de una medida todavía inexplorada como la Renta Básica daría lugar a un nuevo modelo que previsiblemente debería apuntar a sí y cómo quedan solucionados problemas urgentes relacionados con la subsistencia. En lo que toca a los sistemas de 
indicadores estructurales de derechos sociales, cabe integrar el estudio de políticas, estándares, buenas prácticas y derecho comparado, por ejemplo, en el ámbito europeo, así como el seguimiento de garantías judiciales y cuasi judiciales. La crisis financiera ha dejado un impacto estructural que ha afectado a los derechos sociales, al mismo tiempo, ha modificado las expectativas sociales relacionadas con la igualdad, así como la sensibilidad en relación con ella. Bajo ese último presupuesto podríamos sugerir medidas concretas sobre transparencia salarial, básicamente, con una importación de derecho comparado de las medidas tomadas por Islandia, y en menor medida, Alemania o Reino Unido, así las relativas a la claridad retributiva en empresas a partir de un número de trabajadores, bajo el principio de eficiencia, podemos decir, con carácter general que las políticas redistributivas no deben ser centrales sino un complemento de las políticas redistributivas.

Se ha defendido, por último, un modelo que integre los análisis de impacto regulatorio tanto en referencia a los mecanismos de eficacia de ese derecho desde su incorporación legislativa (en términos de mejora de técnica legislativa, con órganos de evaluación, supervisión externa hasta la integración de formularios previos sobre el impacto que tal o cual legislación puede tener sobre la polarización económica, la distribución de la renta o la cohesión social. 


\section{Bibliografía}

ABRAMOVICH, V. y COURTIS, CH., Los derechos sociales en el debate democrático, Madrid: Bomarzo, 2006.

AÑÓN ROIG, M. J., «Derechos sociales: cuestiones de legalidad y de legitimidad», Anales de la Cátedra Francisco Suárez, núm., 44, 2010, pp.15-41.

AÑÓN ROIG, M. J., «¿Hay límites a la regresividad de los derechos sociales?», Derechos y libertades, núm. 34, 2016, pp. 82-83.

AÑÓN ROIG, M. J., «Derechos humanos y deberes: Efectividad y prohibición de regresividad», Repensar los Derechos Humanos / ANGELES RÓDENAS (ed. lit.), 2018, pp. 263-305.

AÑÓN ROIG, M. J., DE LUCAS, J., Integración y derechos: a la búsqueda de indicadores, Icaria, 2013.

ASENSI SABATER, J., «Origen e historia del Estado Social», Lecciones sobre Estado Social y Derechos Sociales / Albert NOGUERA FERNÁNDEZ A., (Dir.), GUAMÁN HERNÁNDEZ A., (Dir.), Valencia: Tirant lo Blanch, 2014, pp. 25-39.

ATKINSON, A. B., Inequality. What can be done, Cambridge: Harvard University Press, 2015.

AUBY, J. B., PERROUD, T. (Eds.), La evaluación de Impacto Regulatorio, Sevilla; Instituto Nacional de Administración Pública y Global Law Press, 2013.

AYALA CAÑÓN, L., «El gasto público en programas de lucha contra la pobreza: tendencias, determinantes y necesidades de reforma», Papeles de economía española, núm. 147, 2016 (Ejemplar dedicado a: Gasto público en España: presente y futuro / coord. por Santiago Lago Peñas, Jorge Martínez Vázquez), pp. 145-166.

AYMERICH, I., Sociología de los derechos humanos. Un modelo weberiano contrastado con investigaciones empiricas, Valencia: Tirant lo Blanch, 2001

AYMERICH, I., «Una contribución para el buen gobierno: sobre las posibilidades de incorporar el análisis de impacto regulatorio al proceso legislativo valenciano, Ética, transparencia, buen gobierno y sistema electoral. Propuestas de mejora de la normativa valenciana, cit., p. 37.

BOBBIO, N., El futuro de la democracia, Barcelona: Plaza y Janés, 1985.

CABO, C., «Teoría crítica de la democracia, en NOGUERA FERNÁNDEZ, A., (Dir.), Crisis de la democracia y nuevas formas de participación, Valencia: Tirant lo Blanch, 2013

CALVO GARCÍA, M., «La implementación y efectividad de los derechos económicos, sociales y culturales», en ZAPATERO GÓMEZ, V., GARRIDO GÓMEZ, M. I.., (Coords.), Los derechos sociales como una exigencia de la justicia, Universidad de Alcalá: Servicio de Publicaciones, 2009, pp. 201-230.

CALVO GARCÍA, M., «Crisis económica y efectividad de los derechos sociales», en BERNUZ M. J., y CALVO, M., (eds.), La eficacia de los derechos sociales, Valencia: Tirant Lo Blanch, 2013, pp. 120 y ss.

CAMPBELL, T., La Justicia. Principales debates contemporáneos, Barcelona: Gedisa, 2002.

CARCELLER, G., «Análisis de impacto normativo. Proceso y metodología en perspectiva autonómica, Ética, transparencia buen gobierno y sistema electoral, Valencia: Tirant lo Blanch, 2019, pp.97-142.

COMISIÓN INTERAMERICANA DE DERECHOS HUMANOS, «Lineamientos para la elaboración de indicadores de progreso en materia de derechos económicos, sociales y culturales», OEA/ser/L/V/II.129, 5 de octubre de 2007. Informes del Observatorio de política social y derechos humanos

FERRAJOLI, L., Derechos y garantías. La ley del más fuerte, Madrid: Trotta, 1999, 
GARCÍA AMADO, J. A., «Impuestos, igualdad y estado social», Derechos y obligaciones en el estado de derecho: actas del III Coloquio Binacional México-España / Abril Uscanga Barradas (Dir. congr.), Jesús Aquilino Fernández Suarez (Dir. congr.), 2017, pp. 679-689, p. 680.

GARCÍA CÍVICO, J., «La (des) igualdad económica y la (in) eficacia social de las normas constitucionales sobre igualdad», Sistema: revista de ciencias sociales, núm. 237, 2015, pp. 91-112.

GARCÍA CÍVICO, J., La integración social del inmigrante a través del Derecho: Hacia un sistema de indicadores, Cuadernos de Democracia y Derechos Humanos $\mathrm{N}^{\circ}$ 4, Universidad Alcalá-Defensor del Pueblo, Alcalá de Henares, Madrid, 2011.

GARCÍA CÍVICO, J., «Indicadores de integración social del inmigrante: el derecho a la educación», Integración y derechos: a la búsqueda de indicadores, DE LUCAS MARTÍN, J., (ed. lit.), AÑÓN ROIG M. J., (ed. lit.), 2013, pp. 37-66.

HACKER, J., PIERSON, P., Let them Eat Tweets: How the Right Rules in an Age of Extreme Inequality, New York, WW Norton \& Co, 2020.

HIERRO, L., La eficacia de las normas jurídicas, Barcelona: Ariel, 2003.

KIRKPATRICK C., PARKER D., Regulatory Impact Assessment Towards Better Regulation?, Chentelham: Edward Eldar Publishing, 2007.

LEMA AÑÓN, C., «Derechos sociales, ¿para quién?: sobre la universidad de los derechos sociales», Derechos y libertades: Revista del Instituto Bartolomé de las Casas, Año no 14, núm. 22, 2010, pp. 179-203.

NOGUERA, J. A., Repensar las politicas sociales: predistribución e inversión social / coord. por Joseba Zalakain, Borja Barragué Calvo, 2017, pp. 207-228

NOGUERA, J. A., «Predistribución: ¿de qué hablamos, y por qué?», El País, 22 de julio de 2015.

NOYA, J., Ciudadanos ambivalentes. Actitudes ante la igualdad y el Estado de bienestar en España, Centro de Investigaciones Sociológicas, 2004. Madrid: Siglo XXI.

MARCOS, F., «Calidad de las normas jurídicas y estudios de impacto normativo», Revista de Administración Pública, $\mathrm{n}^{\circ} .179$, mayo-agosto 2009, p. 336.

PÉREZ LUÑO, A. E., Dimensiones de la igualdad, Madrid: Dykinson, 2005, pp. 15-18.

PIKETTY, T., Capital in the 21st Century, London: The Belknap Press of Harvard University Press, 2014.

PISARELLO, G., Los derechos sociales y sus garantias: elementos para una reconstrucción, Madrid: Trotta, 2007.

ROSANVALLO, P., La sociedad de los iguales, Madrid: RBA, 2012.

SEPÚLVEDA, m., «La interpretación del Comité de Derechos Económicos, Sociales y Culturales de la expresión "progresivamente"», en COURTIS Ch., (Comp.), Ni un paso atrás. La prohibición de regresividad en materia de derechos sociales, CEDAL-CELS, Buenos Aires, 2006, pp. 117-152.

SUSÍN BETRÁN, R., «Derechos sociales-trabajo-ciudadanía-democracia. Una relación necesaria (y conveniente)"», en M. J. BERNUZ y M. CALVO (eds.), La eficacia de los derechos sociales, Valencia: Tirant Lo Blanch, 2013, pp. 163-194.

WILKINSON, R., PIKCKETT, K., Desigualdad: Un Análisis de la (in)felicidad colectiva. Madrid: Turner Publicaciones, 2009. 